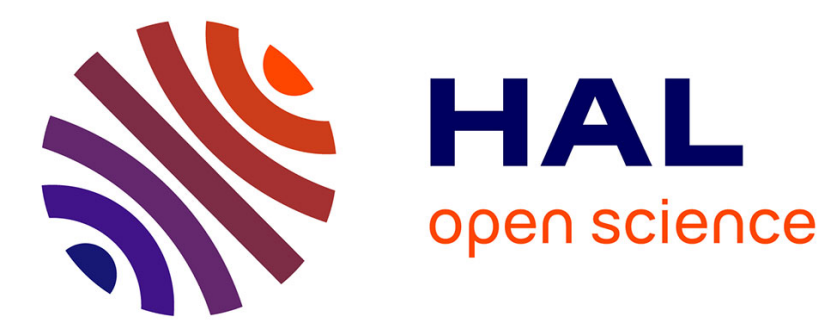

\title{
Efficiency of laminar and turbulent mixing in wall-bounded flows
}

\author{
Benjamin Kadoch, Wouter J.T. Bos, Kai Schneider
}

\section{To cite this version:}

Benjamin Kadoch, Wouter J.T. Bos, Kai Schneider. Efficiency of laminar and turbulent mixing in wallbounded flows. Physical Review E , 2020, 101 (4), 10.1103/PhysRevE.101.043104 . hal-02546585

\section{HAL Id: hal-02546585 \\ https://hal.science/hal-02546585}

Submitted on 18 Apr 2020

HAL is a multi-disciplinary open access archive for the deposit and dissemination of scientific research documents, whether they are published or not. The documents may come from teaching and research institutions in France or abroad, or from public or private research centers.
L'archive ouverte pluridisciplinaire HAL, est destinée au dépôt et à la diffusion de documents scientifiques de niveau recherche, publiés ou non, émanant des établissements d'enseignement et de recherche français ou étrangers, des laboratoires publics ou privés. 


\title{
Efficiency of laminar and turbulent mixing in wall-bounded flows
}

\author{
Benjamin Kadoch, ${ }^{1}$ Wouter J. T. Bos, ${ }^{2}$ and Kai Schneider ${ }^{3}$ \\ ${ }^{1}$ Aix-Marseille Université, IUSTI-CNRS, Marseille, France \\ ${ }^{2}$ LMFA-CNRS-École Centrale de Lyon, France \\ ${ }^{3}$ Aix-Marseille Université, I2M-CNRS, Marseille, France
}

(Received 15 October 2019; accepted 18 March 2020; published 13 April 2020)

\begin{abstract}
A turbulent flow mixes in general more rapidly a passive scalar than a laminar flow does. From an energetic point of view, for statistically homogeneous or periodic flows, the laminar regime is more efficient. However, the presence of walls may change this picture. We consider in this investigation mixing in two-dimensional laminar and turbulent wall-bounded flows using direct numerical simulation. We show that for sufficiently large Schmidt number, turbulent flows more efficiently mix a wall-bounded scalar field than a chaotic or laminar flow does. The mixing efficiency is shown to be a function of the Péclet number, and a phenomenological explanation yields a scaling law, consistent with the observations.
\end{abstract}

DOI: 10.1103/PhysRevE.101.043104

\section{INTRODUCTION}

For a Newtonian fluid, flowing through a pipe of diameter $D$ and length $l$ at a given rate, the pressure drop $\Delta p$ between both ends is a direct measure of the energy injected in the system. A common way to express this is by the friction factor $f$,

$$
f=2 \frac{D}{l} \frac{\Delta p}{\rho U^{2}},
$$

where $\rho$ is the density and $U$ the mean velocity. Whenever the flow transitions to a turbulent state, the power one needs to supply is larger than for a laminar flow. For turbulent flow through smooth pipes, the ratio of energy injected to obtain a given flow rate to the energy needed to obtain the same rate for a laminar flow can be roughly estimated by comparing the Poiseuille solution $f=64 / \mathrm{Re}$ to the turbulence friction factor proposed by Blasius, $f=0.316 \mathrm{Re}^{-1 / 4}$ [1], yielding

$$
\frac{P_{\text {turb }}}{P_{\text {lam }}}=4.9 \times 10^{-3} \mathrm{Re}^{3 / 4},
$$

where $\operatorname{Re}=U D / v$ with $v$ the kinematic viscosity. This ratio can become very large for large values of the Reynolds number. Energetically, turbulence is therefore not a desirable, but in general an inevitable, feature of pipe flow and numerous engineering flows.

In the case of mixing, it is well known that a turbulent flow rapidly mixes a scalar, and often turbulence is a welcome ingredient of a process where efficient mixing is required. However, it is not a priori known whether energetically it is desirable to mix a system in turbulent flow or that a laminar flow will succeed a given level of mixedness using less energy. It was illustrated by Raynal and Gence [2] that from such an energetic viewpoint, laminar mixing is more efficient than turbulent mixing. That study assumed, however, an infinite or periodic domain. In more recent works $[3,4]$ it was illustrated that the presence of solid boundaries can affect the mixing process dramatically. Indeed, it turned out to be of major importance to take into account the fact that unmixed regions near the walls of the domain act like reservoirs from which the scalar is injected into the flow in a fairly continuous matter. It was shown that this effect significantly decreases the effectiveness of a flow to mix. That particular investigation considered the Stokes limit and did not assess the energy balance of the system and answers therefore not the question whether in the case of turbulent flow, the boundaries affect the mixing efficiency in a similar matter, and that is the subject of the present investigation. Indeed, the presence of boundaries importantly affects turbulent flows, since walls act as sources of vorticity in turbulent flows. The role of coherent vortices for mixing was investigated in Ref. [5]. Also, confinement can directly affect the temporal decay rate of scalar variance [6] and so can the nature of the boundary conditions (i.e., slip or no slip) [7]. Moreover, Thiffeault et al. showed in Ref. [8] that the presence of moving walls improves the mixing. A recent review on chaotic mixing can be found in Ref. [9], and a more complete treatise is Ref. [10]. Attempts to integrate the influence of nontrivial boundary conditions on the theoretical description of mixing are presented in Ref. [11], where the authors focused on mixing in a nearwall region. In Ref. [12] a three-dimensional steady mixing flow is constructed contained by no-slip boundaries, which allows us to establish an upper bound for the mixing rate in such flows. In Ref. [13] a Lagrangian approach to study turbulent dissipation is extended to the case in the presence of walls. However, it seems that the case of wall-bounded mixing has received far less interest from a theoretical viewpoint than the unbounded or spatially periodic case has. In the present work we contribute to this subject with observations from, and a quantitative analysis of, well-controlled numerical simulations.

To summarize the question we address, as positioned with respect to existing work: Raynal and Gence [2] showed that in infinite or periodic domains, energetically it is favorable to mix using a laminar flow. Reference [3] showed that the mixing rate is radically changed when a laminar mixing flow 


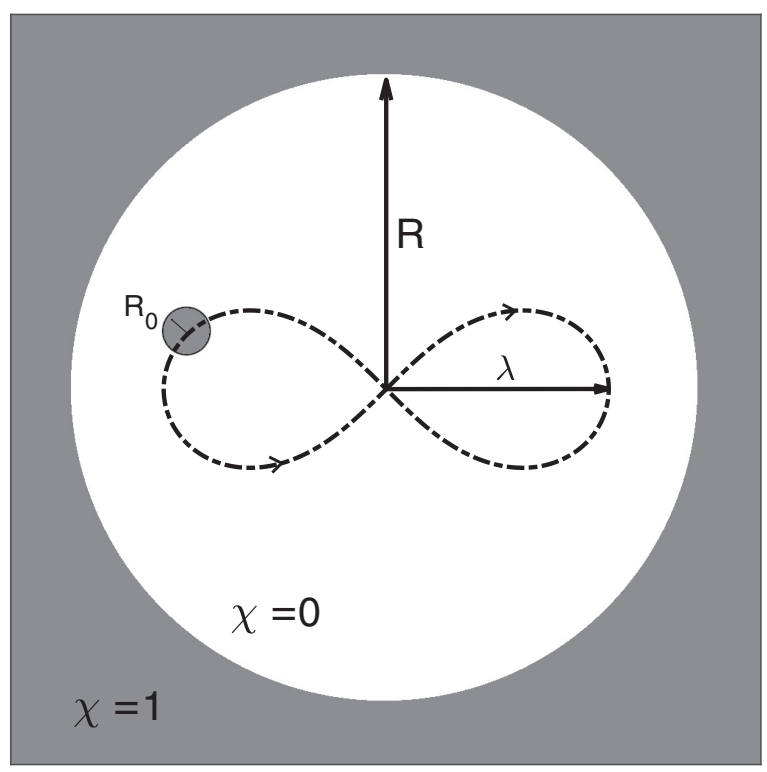

FIG. 1. Sketch of the computational domain and the trajectory of the mixing rod. The white region $(\chi=0)$ corresponds to the fluid, and the gray region $(\chi=1)$ corresponds to the solid part of the domain.

is confined by a solid wall. We will investigate whether in wall-bounded domains it is favorable to mix by a laminar flow or a turbulent flow. We will attempt to propose an expression similar to Eq. (2) to describe our results.

We will consider a simple two-dimensional flow in a circular container with a circular rod, stirring a passive scalar, considering a broad range of values of the Reynolds and Schmidt numbers. The setup is similar to the one investigated experimentally at low Reynolds number in Ref. [3]. A definition of the energetic mixing efficiency is introduced in Sec. II, the setup and the numerical method used are discussed in Sec. III, and the results obtained to quantify the energy efficiency are reported on in Sec. IV. In Sec. V we propose a phenomenological explanation to describe the results. Finally, conclusions and perspectives will be given.

\section{DEFINITION OF AN ENERGETIC MIXING EFFICIENCY}

We consider passive scalar mixing in a circular domain that is stirred with a rod. Figure 1 shows a sketch of the computational domain, a rectangle of size $L_{x} \times L_{y}$. The diameter of the fluid domain which is immersed in the rectangle is $2 R$, and the fluid is set into motion by a circular rod of radius $R_{0}=a R$, describing an eight-shaped motion, parametrized by a lemniscate of Bernoulli similar to [3]

$$
\begin{gathered}
X(t)=X_{0}+\frac{\lambda \sin (t)}{1+\cos (t)^{2}}, \\
Y(t)=Y_{0}+\frac{\lambda \sin (t) \cos (t)}{1+\cos (t)^{2}},
\end{gathered}
$$

where $\left(X_{0}, Y_{0}\right)$ is the center of the domain and $\lambda$ is the length of the lemniscate's half axis. The initial condition of the passive scalar is a Gaussian blob where the phases of the
Fourier coefficients have been randomized and thus the spatial structure has been destroyed. It corresponds to a statistically Gaussian distribution of the scalar. At $t=0$, the rod is set into motion until obtaining a statistically steady state. Once the latter state is accomplished, the scalar is injected into the flow. Its initial volume averaged value $k_{\theta}$ is set to unity.

The Navier-Stokes equations for incompressible flow read

$$
\partial_{t} \boldsymbol{u}+\boldsymbol{u} \cdot \nabla \boldsymbol{u}=-p+\frac{1}{\operatorname{Re}} \Delta \boldsymbol{u}
$$

combined with the continuity equation $\boldsymbol{\nabla} \cdot \boldsymbol{u}=0$, where $\boldsymbol{u}$ is the velocity and $p$ the pressure. The velocity satisfies no-slip and no penetration conditions at the wall. In the limit of very low Reynolds number, the nonlinear term of Eq. (5) becomes negligible, and in this limit the flow becomes purely diffusive. This "Stokes" regime can be described by

$$
\partial_{t} \boldsymbol{u}=-\nabla p+\frac{1}{\mathrm{Re}} \Delta \boldsymbol{u} .
$$

The Reynolds number used here, $\operatorname{Re}=U L / v$, is based on a characteristic length scale $L$ (for convenience we choose $L=$ 1 in the following) and the speed $U$ of the stirring rod. The geometry is determined not only by the size of the domain, but also by the rod size and the radius $\lambda$ of the lemniscate. We have not varied these parameters and have used constant values for the geometrical parameters.

The dynamics of a passive scalar $\theta$ is governed by an advection-diffusion equation,

$$
\partial_{t} \theta+\boldsymbol{u} \cdot \nabla \theta=\frac{1}{\mathrm{Pe}} \Delta \theta,
$$

where the Péclet number is $\mathrm{Pe}=U L / \alpha$, with $\alpha$ the diffusivity. No-flux of the passive scalar is imposed at the wall. A useful dimensionless number is the Schmidt number $\mathrm{Sc}=v / \alpha$, comparing diffusivity and kinematic viscosity. The dimensionless numbers are related via $\mathrm{Pe}=\mathrm{Re} S \mathrm{Sc}$.

The main question in this study is how much energy it costs to mix the scalar. The kinetic energy in the system is

$$
k(t)=\frac{1}{2} \int_{V}\|\boldsymbol{u}\|^{2} d V
$$

and the scalar variance

$$
k_{\theta}(t)=\frac{1}{2} \int_{V} \theta^{2} d V
$$

where $V$ is the volume of the fluid domain. The evolution of $k$ and $k_{\theta}$ is given by

$$
\begin{gathered}
\frac{d k(t)}{d t}=P_{i n}(t)-\epsilon(t), \\
\frac{d k_{\theta}(t)}{d t}=-\epsilon_{\theta}(t),
\end{gathered}
$$

where the dissipation of kinetic energy and of scalar fluctuations are, respectively, defined as

$$
\begin{gathered}
\epsilon(t)=\frac{1}{\operatorname{Re}} \int_{V} \omega(t)^{2} d V, \\
\epsilon_{\theta}(t)=\frac{1}{\operatorname{Pe}} \int_{V}\|\nabla \theta(t)\|^{2} d V .
\end{gathered}
$$


Note that expression (12) is specific to this two-dimensional case, where the vorticity $\omega$ reduces to a scalar quantity. The passive scalar variance equation does not contain a source term, and since the dissipation is a positive quantity, the variance decays monotonically from a given initial value. The kinetic energy balance contains the energy injection by the moving rod, represented by $P_{i n}$. It is this injected energy which interests us. The cumulative total injected energy is obtained by integrating Eq. (10),

$$
\Pi(t)=\int_{0}^{t} P_{i n}(\tau) d \tau=k(t)+\int_{0}^{t} \epsilon(\tau) d \tau .
$$

In a Stokes flow, $\Pi(t)$ will be a monotonically growing function of time. In a turbulent flow there will also be a growing trend, but fluctuations around it can be observed, reflecting the fluctuations of the kinetic energy in the domain. In both the Stokes and turbulent cases, the total injected energy will tend to infinity at infinite times. To estimate the energy needed to mix we need to define a criterion. Our criterion for the mixing time $\tau_{\beta}$ is

$$
\frac{k_{\theta}\left(t=\tau_{\beta}\right)}{k_{\theta}(t=0)}=\beta,
$$

where $\beta \in[0,1]$ measures the amount of remaining scalar variance. For instance, $\tau_{0.01}$ corresponds to the time at which the variance is reduced to $1 \%$ of its initial value. Subsequently we introduce the amount of injected energy at time $\tau_{\beta}$ :

$$
\Pi_{\beta}=\Pi\left(t=\tau_{\beta}\right) .
$$

The quantity $\Pi_{\beta}$ is thus a measure for the required energy to mix a scalar and to reduce the initial variance to a fraction $\beta$.

In the following, we will compare the quantity $\Pi_{\beta}$ for different values of $\beta, \operatorname{Re}$, and Sc, and we will assess how $\Pi_{\beta}$ depends on the different quantities.

\section{SETUP AND NUMERICAL METHOD}

The numerical implementation is a Cartesian Fourier pseudospectral method, combined with a volume-penalization technique to model the presence of the circular boundaries and the rod. Time-integration is performed by a secondorder Adams-Bashforth scheme. Details of the code are given in Ref. [14], and its recent implementation in the FLUSI framework is described in Ref. [15]. The volume-penalization method was proposed by Ref. [16] for imposing no-slip boundary conditions in fluid flow. It was extended to no-flux conditions in Ref. [17] for passive scalars, and Ref. [18] presents an extension for moving objects. These methods treat boundaries by an immersed boundary principle, where the solid boundaries are imposed using a forcing term in the evolution equations. We consider here no-slip boundary conditions for the velocity and no-flux conditions for the passive scalar. The size of the domain is $L_{x} \times L_{y}$ where $L_{x}=$ 24 and $L_{y}=24$. The diameter of the circular domain and of the rod is $R=10$ and $R_{0}=0.8$, respectively. The motion of the rod center follows the parametric equations of a lemniscate of Bernoulli with $\lambda=7.12$, and constant speed $U=2$. The characteristic length $L$ is chosen to be equal unity for convenience of the logarithmic plots. The spatial resolution

\begin{tabular}{|c|c|c|c|c|c|}
\hline $\mathrm{Sc}$ & $\mathrm{St}$ & $10^{1}$ & $10^{2}$ & $10^{3}$ & $10^{4}$ \\
\hline $10^{0}$ & $\checkmark$ & $\checkmark$ & $\checkmark$ & $\checkmark$ & $\checkmark$ \\
\hline $10^{1}$ & $\checkmark$ & $\checkmark$ & $\checkmark$ & $\checkmark$ & \\
\hline $10^{2}$ & $\checkmark$ & $\checkmark$ & $\checkmark$ & & \\
\hline $10^{3}$ & $\checkmark$ & $\checkmark$ & & & \\
\hline $10^{4}$ & $\checkmark$ & & & & \\
\hline
\end{tabular}

TABLE I. Summary of the simulations for different Schmidt and Reynolds numbers. St denotes the Stokes regime.

is $N=1024^{2}$ and the time step is $d t=10^{-4}$ such that the stability constraint of the explicit time scheme is satisfied.

Specifically, the vorticity field $\omega=\boldsymbol{\nabla} \times \boldsymbol{u}$ is computed by

$$
\partial_{t} \omega+\boldsymbol{u} \cdot \nabla \omega=\frac{1}{\operatorname{Re}} \Delta \omega-\nabla \times\left(\frac{\chi}{\eta}\left[\boldsymbol{u}-\boldsymbol{u}_{s}\right]\right) .
$$

where $\chi$ is the mask function, which equals 1 inside the solid and 0 inside the fluid region. Note that in practice we have two mask functions, one for the outer cylinder wall for which $\boldsymbol{u}_{s}=0$ and $\chi$ being time independent, and one for the rod. The latter is time-dependent, i.e., its center follows the trajectory of the lemniscate with a constant velocity norm $\left\|\boldsymbol{u}_{s}(t)\right\|=U$ to impose no-slip conditions of the rod. The penalization parameter $\eta$ corresponds to the permeability of the solid. A small value $\eta=10^{-4}$ is imposed to limit the method's error [16] for the given spatial resolution [15].

To study scalar mixing, we use the penalization technique for the advection-diffusion equation:

$$
\begin{aligned}
\partial_{t} \theta & +\left[(1-\chi) \boldsymbol{u}+\chi \boldsymbol{u}_{s}\right] \cdot \boldsymbol{\nabla} \theta \\
& =\nabla \cdot\left\{\left[\frac{1}{\mathrm{Pe}}(1-\chi)+\varepsilon \chi\right] \nabla \theta\right\},
\end{aligned}
$$

where $\theta$ is the passive scalar. The penalization parameter $\varepsilon$ for the passive scalar equation is chosen sufficiently small according to Ref. [17] and imposes the ratio between the diffusivity and the penalization parameter to be constant with $\alpha / \varepsilon=10^{-9}$.

\section{MAIN RESULTS}

We report here on 15 simulations we carried out, for Schmidt numbers in the range $\left[1,10^{4}\right]$ and Reynolds numbers in the range $\left[1,10^{4}\right]$, in addition to simulations of Stokes flow, where the nonlinear term in the Navier-Stokes equations is set strictly to zero. Also in this case we need to define the visocity and have set its value to unity, corresponding to a Reynolds number of order unity. This Reynolds number does therefore not quantify the ratio of inertial to viscous forces, but quantifies the typical timescale of the viscous diffusion compared to the stirring frequency. The limitation of the attainable numerical grid resolution restricts the Péclet number to be Pe $\leqslant 10^{4}$, which implies that for increasing Schmidt number the Reynolds number needs to be reduced, and vice versa (see Table I). 


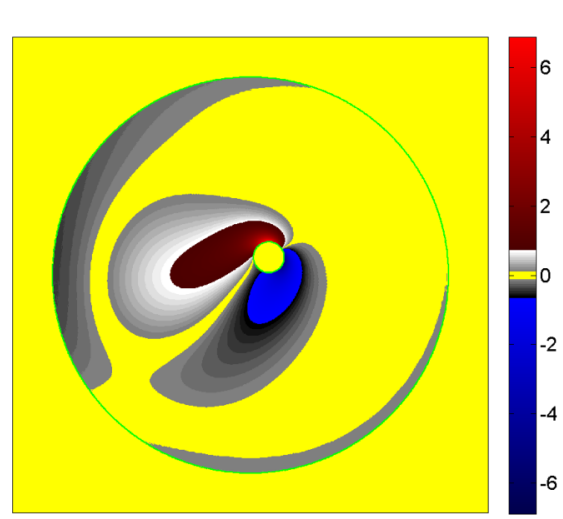

(a)

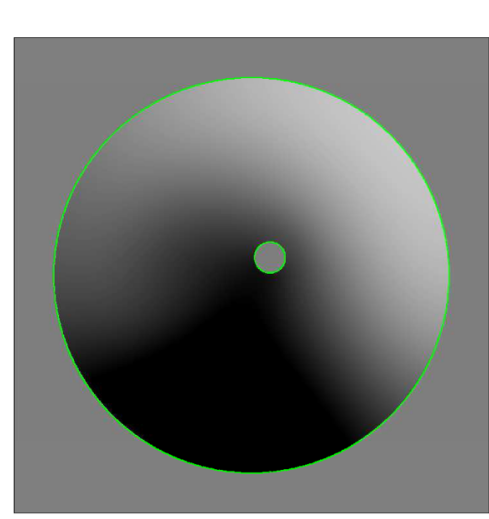

(b)
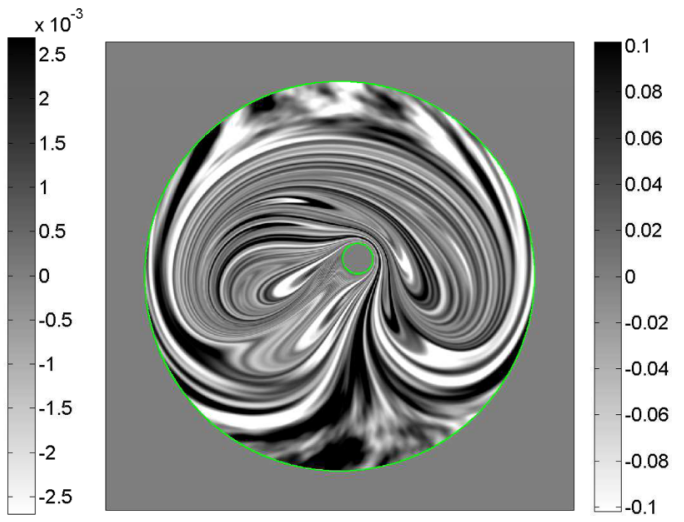

(c)

FIG. 2. Visualizations of the vorticity field (a), the scalar field for Sc $=1$ (b) and the scalar field for Sc $=10000$ (c) for the Stokes flow simulations, at time $t=50$. Min and max values of the colorbar for scalar fields correspond to $\pm 2 \sigma_{\theta}$, where $\sigma_{\theta}$ is the standard deviation of the scalar $\theta$.

\section{A. Flow characterization \\ 1. Stokes flow}

Figure 2(a) shows a visualization of the vorticity field generated by the moving rod in Stokes flow. The main vorticity generation is situated on the rod surface. In Figs. 2(b) and 2(c) we visualize the scalar field at $t=50$ for Schmidt numbers 1 and $10^{4}$, respectively. The most important remark, with respect to mixing, is that most of the unmixed fluid is close to the wall for $\mathrm{Sc}=10^{4}$. This explained the observations of slow mixing in investigation [3] for a similar case. For $\mathrm{Sc}=1$ this unmixed scalar is less present since the diffusive mixing contributes substantially to the overall scalar mixing at low values of Sc.

Figure 3 presents the evolution of the scalar variance as a function of time for values of the Schmidt number ranging from 1 up to $10^{4}$. The general trend is that mixing is slower for increasing values of the Schmidt number. Indeed, for large $\mathrm{Sc}$ the unmixed fluid close to the wall diffuses less towards the center of the domain, which implies that the overall variance

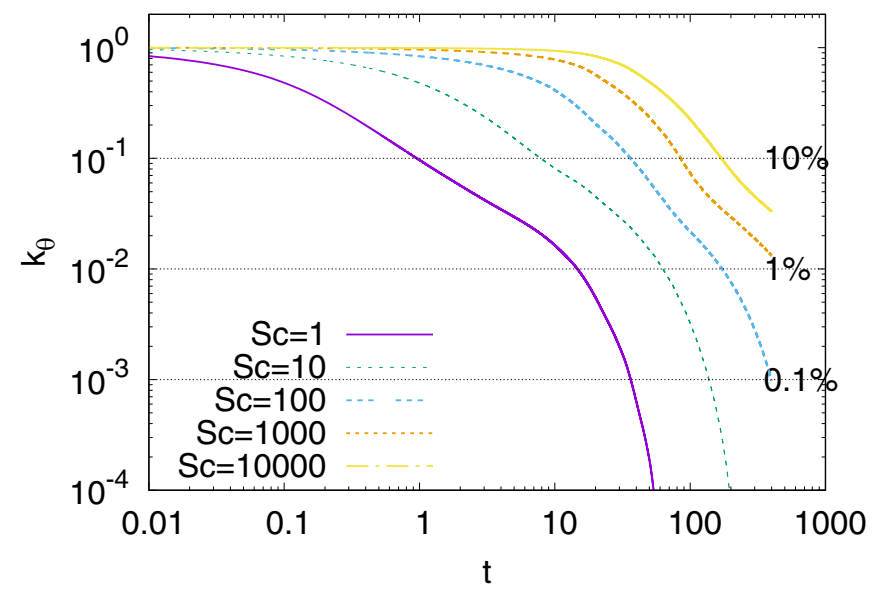

FIG. 3. Time evolution of the variance of passive scalar in the Stokes flow, for different values of the Schmidt number. Horizontal dashed lines indicate three distinct levels of mixedness $\beta=0.1,0.01$ and 0.001 . decays more slowly. The dashed horizontal lines correspond to three levels of mixedness, respectively, when the variance is reduced to a fraction $\beta=0.1,0.01$, and 0.001 of its initial value. For the highest values of $\mathrm{Sc}$, the increasing time of the simulations does not allow us to reach the largest levels of mixedness.

\section{Flow at higher Reynolds number}

The main difference, when the inertial term in the NavierStokes equations is not neglected, is that vorticity detaches from the region where it is generated. This is illustrated in Fig. 4, where circular vortices are clearly observed in the entire domain for $\operatorname{Re}=10^{3}$. These vortices interact with each other, but, more important, penetrate the domain close to the wall, where they allow an efficient mixing in the part of the flow which is not easily mixed in the Stokes regime.

In Figs. 5(a) and 5(b) we show the evolution of the scalar variance as a function of time for values of the Schmidt number ranging from 1 up to $10^{4}$. The different levels of mixedness are again indicated by dashed lines. A larger value of Sc leads also to a slower mixing.

In particular in Fig. 5(b), it is interesting to note that at short times for a given $\mathrm{Sc}$, the Stokes flow mixes most efficiently, whereas at longer times, the highest Reynolds number flow attains the thresholds of mixedness $\beta=0.01$ and $\beta=0.001$ before the other flows do. Clearly the molecular diffusion is at longer times less efficient than the mixing induced by vortical structures. What we do not know yet is if it is also energetically desirable to use turbulent mixing and that will be determined now.

\section{B. Mixing efficiency}

We have evaluated the mixing times $\tau_{\beta}$ for different values of $\beta=0.1,0.01,0.001$, and summarize them in Fig. 6 for all the different values of $\mathrm{Re}$ and Sc. The general trend is that the mixing time increases with $\mathrm{Sc}$ and decreases with Re. Clearly stirring the fluid faster mixes the scalar more rapidly. However, does a higher Reynolds number also increase the efficiency in terms of injected energy? We recall that for unbounded flows it was shown that this is not the case [2]. 


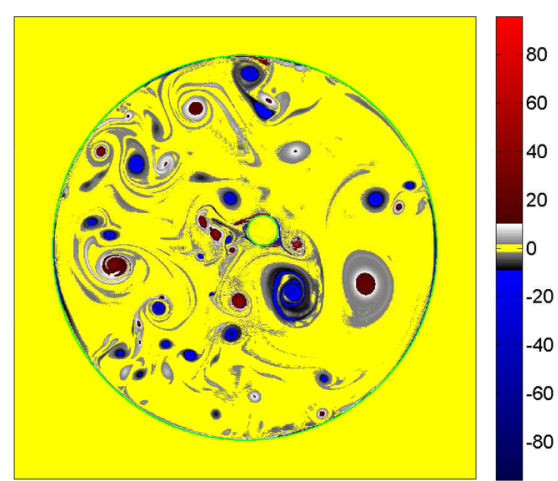

(a)

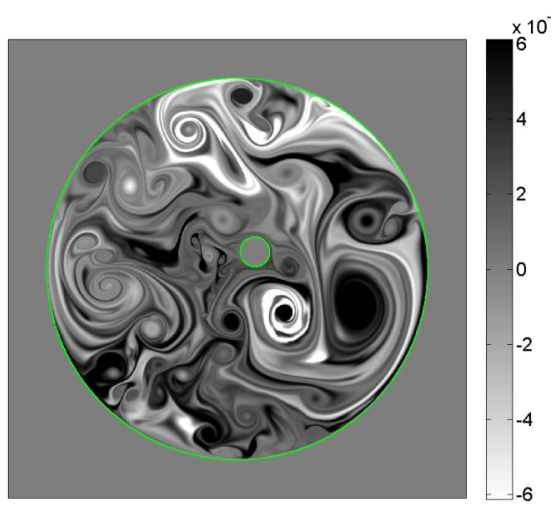

(b)
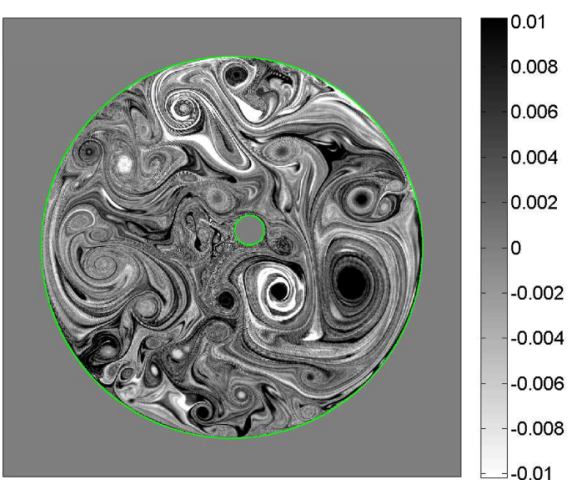

(c)

FIG. 4. Visualizations of the vorticity field (a), the scalar field for $\mathrm{Sc}=1$ (b) and the scalar field for $\mathrm{Sc}=10$ (c) for the simulations with $\operatorname{Re}=1000$ at time $t=50$. Min and max values of the color bar for scalar fields correspond to $\pm 2 \sigma_{\theta}$, where $\sigma_{\theta}$ is the standard deviation of the scalar $\theta$. The thin green line indicates the interface between the fluid and solid domain.

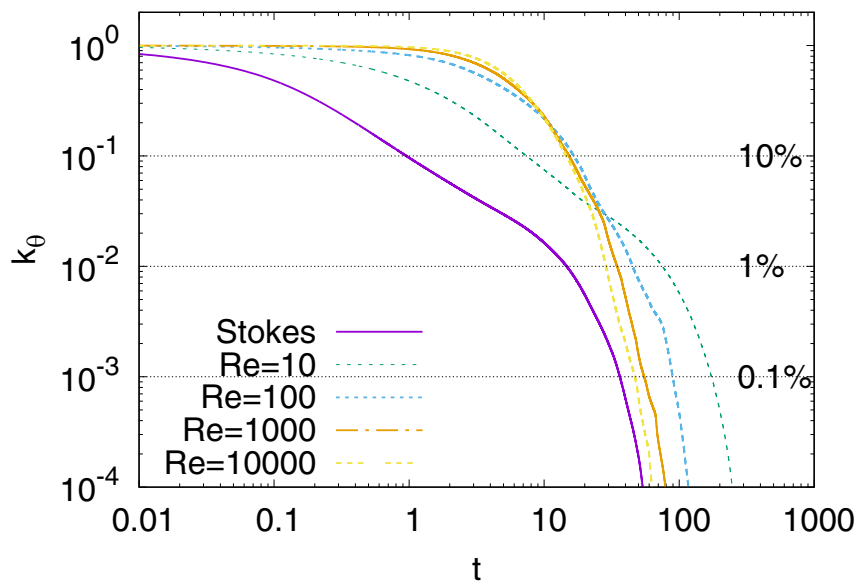

(a)

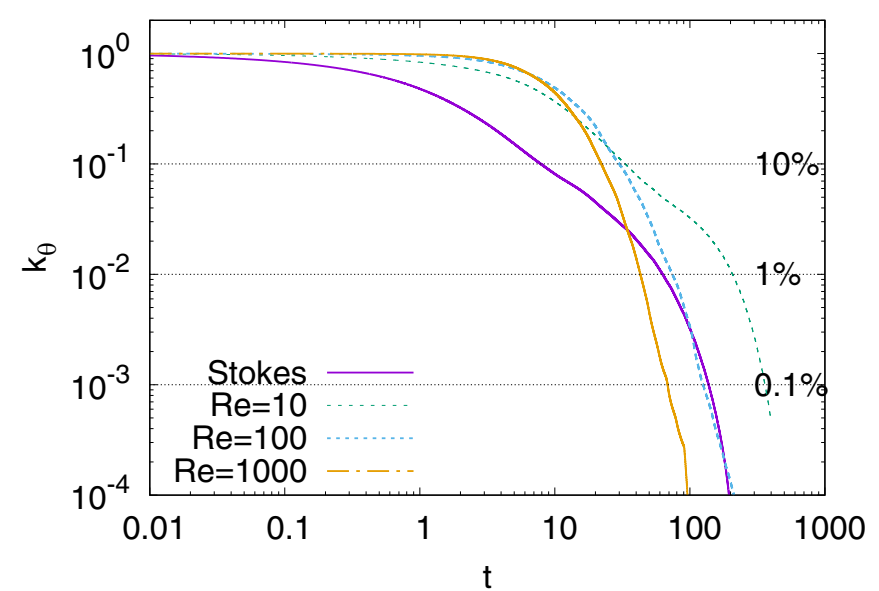

(b)

FIG. 5. Time evolution of the variance of passive scalar for different values of the Reynolds and Schmidt numbers; Sc $=1$ (a) and Sc $=10$ (b). Horizontal dashed lines indicate three distinct levels of mixedness: $\beta=0.1,0.01$, and 0.001 .

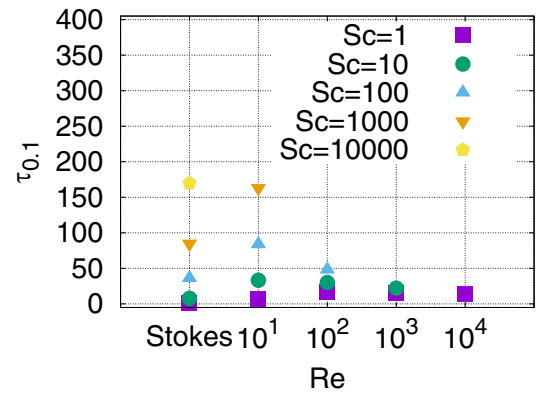

(a)

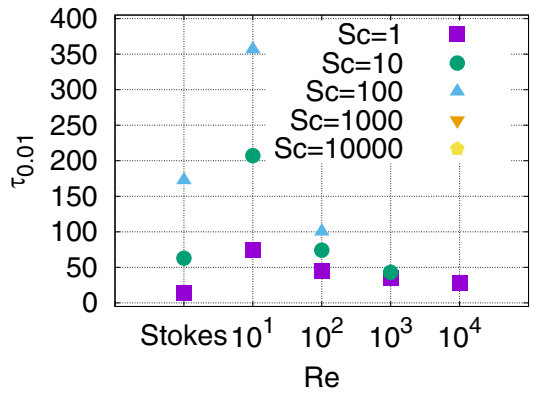

(b)

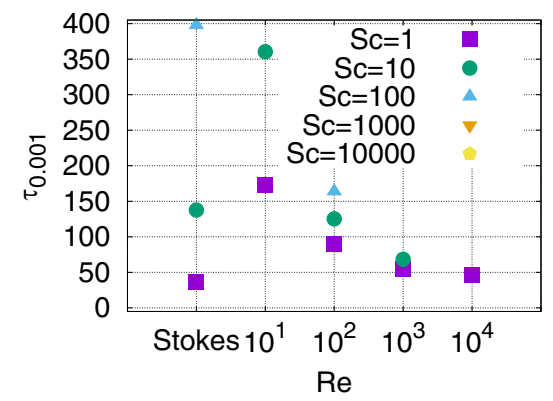

(c)

FIG. 6. Mixing times $\tau_{\beta}$, corresponding to the time it takes for the flow to decrease the value of scalar variance to a fraction $\beta$ of its initial value. 


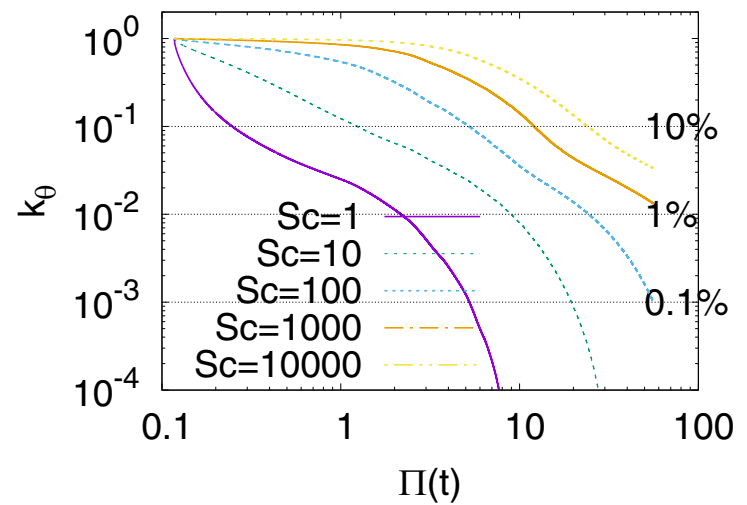

(a)

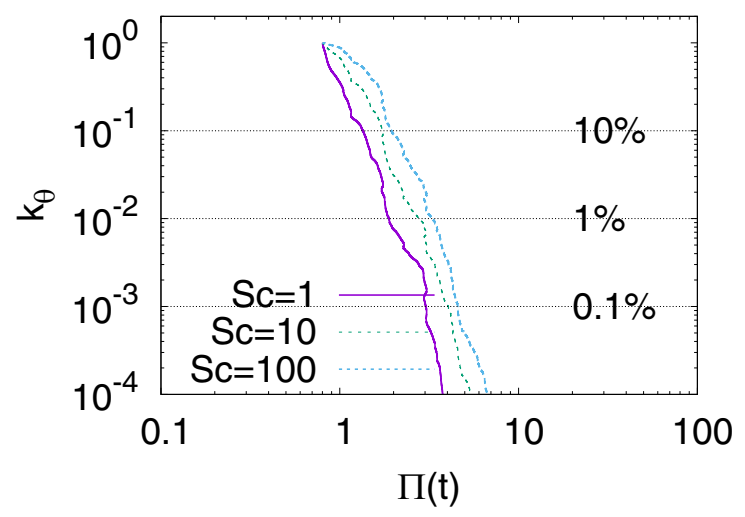

(c)

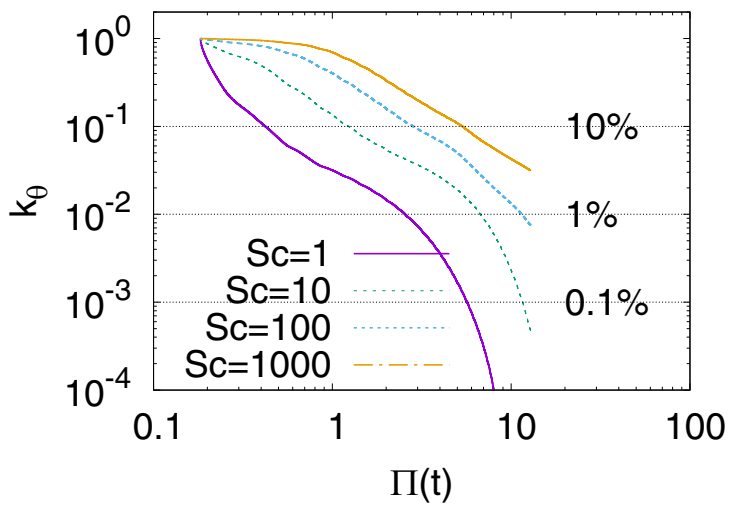

(b)

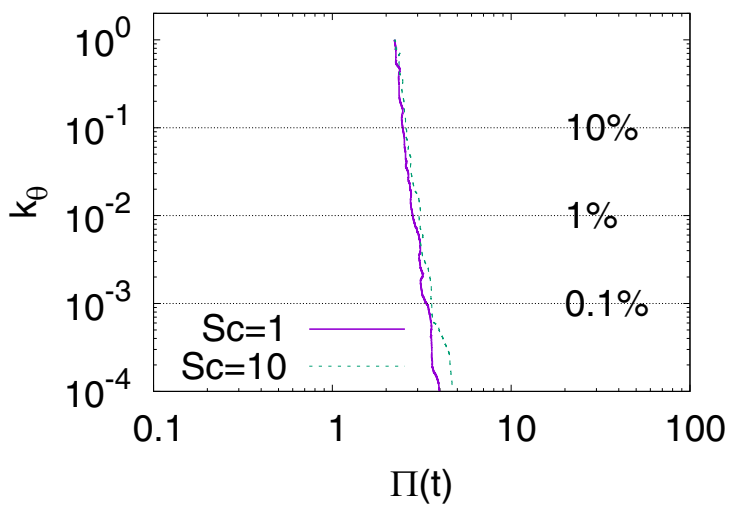

(d)

FIG. 7. Parametric plot of the evolution of the variance of passive scalar for different Reynolds number as a function of the injected energy $\Pi(t)$, for different values of the Schmidt number. (a) Stokes flow; (b) $\operatorname{Re}=10$; (c) $\operatorname{Re}=100$; (d) $\operatorname{Re}=1000$. Horizontal dashed lines indicate three distinct levels of mixedness.

In Figs. 7 and 8 we plot the evolution of the variance of the scalar, but this time as a function of the injected energy $\Pi(t)$ instead of time. The decay is not strictly monotonous because of the fluctuations of the kinetic energy, but the global trend of $k_{\theta}(\Pi)$ is decaying for increasing injected energy. Again we indicate the three levels of mixedness by horizontal dashed lines. The values of $\Pi_{\beta}$ are summarized in Fig. 9.
This figure clearly shows that the needed amount of energy to achieve a fixed decrease of the variance is a decreasing function of the Péclet number. The answer to the question we asked - "is it energetically favorable to stir a confined scalar flow strong enough to make the flow turbulent?"- can thus be answered positively. This disagrees with theoretical results for unbounded flows [2], but can be understood in the light

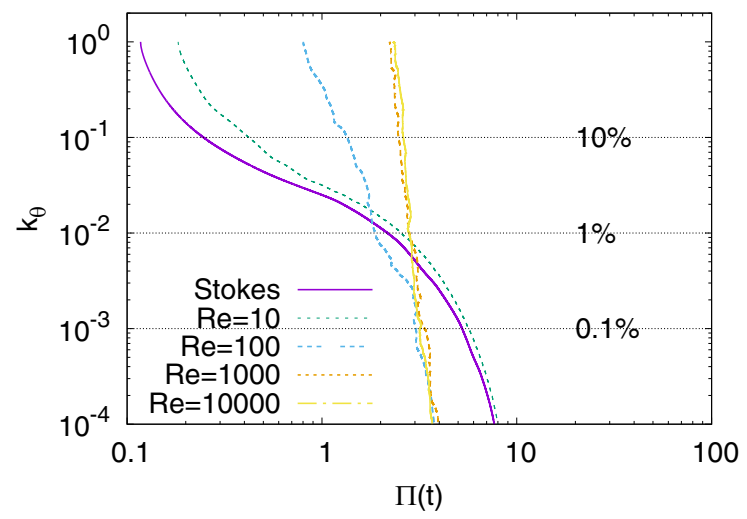

(a)

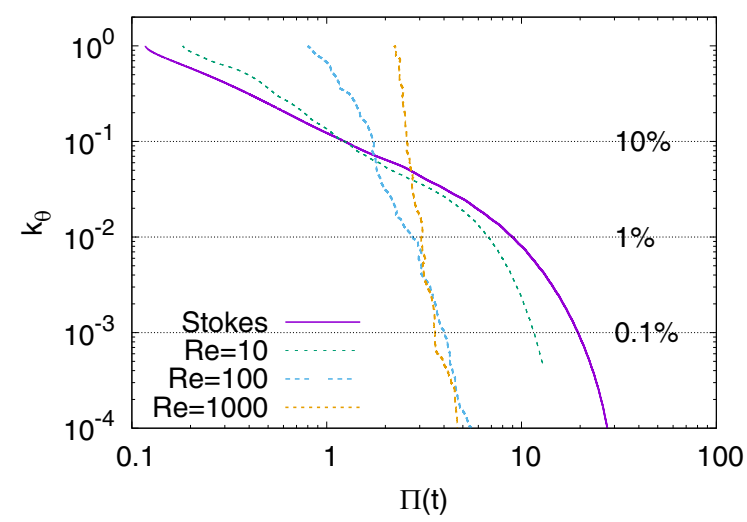

(b)

FIG. 8. Parametric plot of the evolution of the variance of passive scalar for different values of the Reynolds number as a function of the injected energy $\Pi(t)$. (a) $\mathrm{Sc}=1$ (b) $\mathrm{Sc}=10$. Horizontal dashed lines indicate three distinct levels of mixedness $\beta=0.1 \%, 1 \%$, and $10 \%$. 


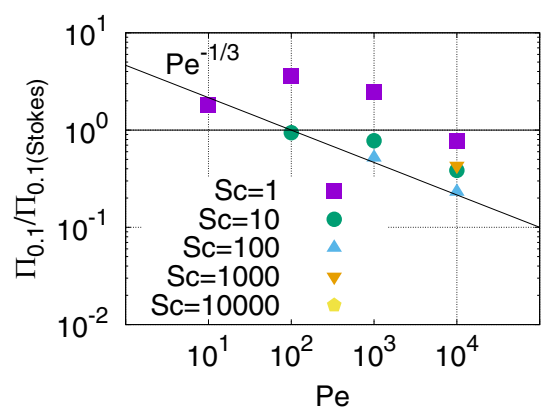

(a)

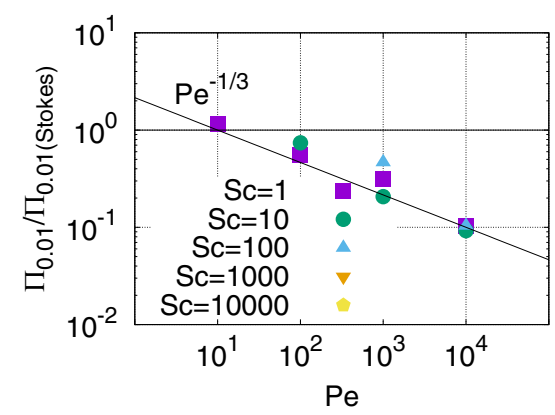

(b)

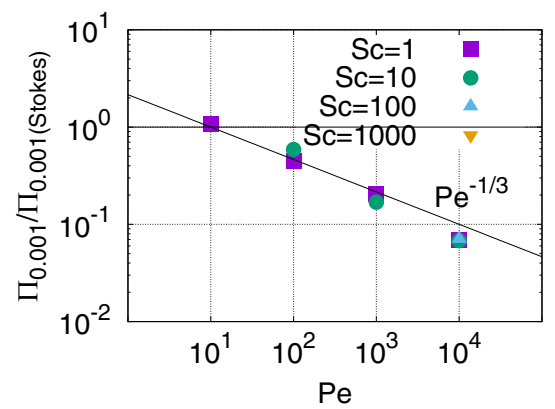

(c)

FIG. 9. Mixing energy $\Pi_{\beta}$, corresponding to the amount of energy injected into the flow at $t=\tau_{\beta}$ plotted as a function of Pe for different Sc numbers, and for (a) $\beta=0.1$; (b) $\beta=0.01$; and (c) $\beta=0.001$. This quantity represents the mixing efficiency of a flow from an energetic viewpoint.

of the observations of chaotic mixing [3] in wall-bounded flow.

\section{PHENOMENOLOGICAL EXPLANATION}

In Fig. 9, in particular in Figs. 9(b) and 9(c), it is observed that the amount of energy needed to attain a level of mixedness compared to the Stokes case is given by a power law of the Péclet number. More precisely the observed relation is close to

$$
\Pi / \Pi_{\text {Stokes }} \sim \mathrm{Pe}^{-1 / 3} .
$$

This is the mixing equivalent of expression (2), which compares the friction in turbulent pipe flow compared to its laminar value. In the present case, the exponent is however negative. This expression can be obtained by dimensional arguments as follows.

It was shown in the case of flux-line expulsion in liquidmetal flows $[19,20]$ and in the case of mixing of a passive scalar $[21,22]$, that the typical mixing timescale $T_{\theta}$ in a flow with a typical velocity-gradient timescale $T_{u}$ is given by

$$
T_{\theta} \sim T_{u} \mathrm{Pe}^{1 / 3} .
$$

This expression holds for mixing in laminar flow. Also in laminar tube flows of diameter $D$ and length $l$ the transfer of heat, characterized by the Nusselt number $\mathrm{Nu}$, is well described by a relation proposed by Sieder and Tate [23,24], writing for constant viscosity,

$$
\mathrm{Nu} \sim \frac{D}{l}(\mathrm{Pe})^{-1 / 3} .
$$

The mixing efficiency in passive scalar mixing is proportional to the inverse of the scalar timescale $T_{\theta}$ (see, for instance, Ref. [25]), and the injected energy to mix is in the Stokes case directly proportional to the time $T_{\theta}$. Therefore we have

$$
\Pi_{\text {Stokes }} \sim \mathrm{Pe}^{1 / 3} .
$$

The phenomenology changes radically when inertia starts to dominate the flow. From Fig. 7 we observe that at high Péclet numbers, the mixing becomes almost independent of the amount of injected energy [the almost vertical drop in
Figs. 7(c) and 7(d) illustrates this]. Therefore, at large values of the Péclet number, when compared to stokes flow, we obtain Eq. (19). These arguments yield a result which describes our data reasonably well. Clearly, further analytical, phenomenological, numerical, or experimental work can help to further assess the validity of the above expression.

\section{CONCLUSIONS AND PERSPECTIVES}

We showed that turbulence increases the mixing efficiency in wall-bounded two-dimensional flows, in comparison to the Stokes regime. The dynamical difference between turbulent and laminar mixing is that in the Stokes case the vorticity equation reduces to a pure diffusion equation, whereas in the nonlinear regime vorticity generated at the boundaries or impellers can detach and can be advected into the entire flow.

This is of crucial importance for mixing. Indeed, the fact that vortices can detach, enter, or leave the near-wall region allows an efficient sweeping and mixing of the near-wall scalar field, penetrating also in regions which would be poorly mixed in the diffusive case. Turbulence is therefore not in all applications hostile to energy savings, and improved mixing can be obtained for large Pe.

It is interesting in the light of the present investigation to mention the recent study [26], where optimization algorithms are applied to determine the most efficient stirring protocols in a setup similar to the one in the present study. In their work, the authors successfully optimize the mixing speed, changing the rod shape and the trajectory. They have not yet considered the most energetic way to mix. To optimize, energetically, the mixing efficiency using their control strategy would certainly be an interesting topic for future work. We think that the overall conclusions of the present investigation are robust, in that turbulent mixing is more efficient than mixing in the Stokes regime, but that changing the details of the geometry and the rod trajectory can certainly lead to a more optimal mixing.

\section{ACKNOWLEDGMENTS}

Centre de Calcul Intensif d'Aix-Marseille is acknowledged for granting access to its high performance computing resources. K.S. thanks the French-Italian LIA "LYSM" (AMU- 
CNRS-ECM and INdAM) and the French Research Federation for Fusion Studies within the framework of the Euro- pean Fusion Development Agreement (EFDA) for financial support.
[1] H. Blasius, Das Ähnlichkeitsgesetz bei Reibungsvorgängen in Flüssigkeiten, in Mitteilungen über Forschungsarbeiten auf dem Gebiete des Ingenieurwesens (Springer, Berlin, Heidelberg, 1913), pp. 1-41.

[2] F. Raynal and J.-N. Gence, Energy saving in chaotic laminar mixing, Int. J. Heat Mass Transf. 40, 3267 (1997).

[3] E. Gouillart, N. Kuncio, O. Dauchot, B. Dubrulle, S. Roux, and J.-L. Thiffeault, Walls Inhibit Chaotic Mixing, Phys. Rev. Lett. 99, 114501 (2007).

[4] E. Gouillart, O. Dauchot, B. Dubrulle, S. Roux, and J.-L. Thiffeault, Slow decay of concentration variance due to no-slip walls in chaotic mixing, Phys. Rev. E 78, 026211 (2008).

[5] C. Beta, K. Schneider, M. Farge, and H. Bockhorn, Numerical study of mixing of passive and reactive scalars in twodimensional turbulent flows using orthogonal wavelet filtering, Chem. Eng. Sci. 58, 1463 (2003).

[6] G. Lamaison, W. J. T. Bos, L. Shao, and J.-P. Bertoglio, Decay of scalar variance in isotropic turbulence in a bounded domain, J. Turbulence 8, N4 (2007).

[7] H. Salman and P. H. Haynes, A numerical study of passive scalar evolution in peripheral regions, Phys. Fluids 19, 067101 (2007).

[8] J.-L. Thiffeault, E. Gouillart, and O. Dauchot, Moving walls accelerate mixing, Phys. Rev. E 84, 036313 (2011).

[9] H. Aref, J. R. Blake, M. Budišić, S. S. S. Cardoso, J. H. E. Cartwright, H. J. H. Clercx, K. El Omari, U. Feudel, R. Golestanian, E. Gouillart, G. F. van Heijst, T. S. Krasnopolskaya, Y. Le Guer, R. S. MacKay, V. V. Meleshko, G. Metcalfe, I. Mezić, A. P. S. de Moura, O. Piro, M. F. M. Speetjens, R. Sturman, J.-L. Thiffeault, and I. Tuval, Frontiers of chaotic advection, Rev. Mod. Phys. 89, 025007 (2017).

[10] J. M. Ottino, The Kinematics of Mixing: Stretching, Chaos, and Transport, Vol. 3 (Cambridge University Press, Cambridge, 1989).

[11] V. V. Lebedev and K. S. Turitsyn, Passive scalar evolution in peripheral regions, Phys. Rev. E 69, 036301 (2004).

[12] R. S. MacKay, A steady mixing flow with no-slip boundaries, in Chaos, Complexity And Transport: Theory and Applications (World Scientific, Singapore, 2008), pp. 55-68.
[13] T. D. Drivas and G. L. Eyink, A Lagrangian fluctuationdissipation relation for scalar turbulence. Part II. Wall-bounded flows, J. Fluid Mech. 829, 236 (2017).

[14] K. Schneider, Numerical simulation of the transient flow behavior in chemical reactors using a penalization method, Comput. Fluids 1223, 006 (2005).

[15] T. Engels, D. Kolomenskiy, K. Schneider, and J. Sesterhenn, Flusi: A novel parallel simulation tool for flapping insect flight using a Fourier method with volume penalization, SIAM J. Sci. Comput. 38, S3 (2016).

[16] P. Angot, C. H. Bruneau, and P. Fabrie, A penalization method to take into account obstacles in viscous flows, Numer. Math. 497, 1999 (1999).

[17] B. Kadoch, D. Kolomenskiy, K. Schneider, and P. Angot, A volume penalization method for incompressible flows and scalar advection-diffusion with moving obstacles, J. Comput. Phys. 231, 4365 (2012).

[18] D. Kolomenskiy and K. Schneider, A Fourier spectral method for the Navier-Stokes equations with volume penalization for moving solid obstacles, J. Comput. Phys. 228, 5687 (2009).

[19] N. O. Weiss, The expulsion of magnetic flux by eddies, Proc. Royal Soc. London. Series A. Mathematical and Physical Sciences 293, 310 (1966).

[20] H. K. Moffatt and H. Kamkar, On the time-scale associated with flux expulsion, in Stellar and Planetary Magnetism, edited by A. M. Soward (Gordon \& Breach, Budapest, 1983), pp. 91-97.

[21] P. B. Rhines and W. R. Young, How rapidly is a passive scalar mixed within closed streamlines? J. Fluid Mech. 133, 133 (1983).

[22] P. Flohr and J. C. Vassilicos, Accelerated scalar dissipation in a vortex, J. Fluid Mech. 348, 295 (1997).

[23] E. N. Sieder and G. E. Tate, Heat transfer and pressure drop of liquids in tubes, Ind. Eng. Chem. 28, 1429 (1936).

[24] A. Bejan and A. D. Kraus, Heat Transfer Handbook, Vol. 1 (John Wiley \& Sons, Inc., Hoboken, New Jersey, 2003).

[25] Y. Yang, R. Chahine, R. Rubinstein, and W. Bos, Passive scalar mixing in modulated turbulence, Fluid Dyn. Res. 51, 045501 (2019).

[26] M. F. Eggl and P. J. Schmid, A gradient-based framework for maximizing mixing in binary fluids, J. Comput. Phys. 368, 131 (2018). 